\title{
Holder valuation and holder rigidity for right ring of fractions
}

\author{
Abdolghafoor Allahyari and Mohammad Hossein Hosseini \\ Department of Mathematics, Farhangian University, Birjand, Iran
}

Received: 8 October 2015, Accepted: 13 July 2016

Published online: 16 August 2017.

\begin{abstract}
The purpose of this article is to introduce the notion of $\left(C_{1}, C_{2}\right)$-Hölder Krull valuation on right ring of fractions ( with respect to right denominator set $S$ in a ring $R$ ). It is proved that if $R$ is a ring satisfying in Hölder rigidity condition, and $S$ a right permutable set of regular elements in $R$, then the right ring of fractions $R^{\prime}=Q_{\varphi}^{r}(R)$ with respect to $S$ satisfies in Hölder rigidity condition. This results provide an extension of the Garsia theorem (see [2]) for right ring of fractions.
\end{abstract}

Keywords: valuation, Hölder valuation, Hölder equivalent, right ring of fractions.

\section{1 introduction and preliminaries}

The theory of valuations may be viewed as a branch of topological algebra. The devolopment of valuation theory has spanned over more than a hundred years. First the notion of valuations on fields was introduced. Details of valuations on fields can be found in many monographs, e. g. Endler (see [1]), Ribenboim (see [7]), and Schilling (see [8]). Then Manis introduced the notion of valuations in the category of commutative rings and it can be found in Manis (see [6]), Huckaba (see [3]), and Knebusch and Zhang (see [4]). A group $\Gamma$ is called an ordered multiplicative group if it has a total ordering $\leq$ which is compatible with the group structure, i. e. $\alpha \leq \beta(\alpha, \beta \in \Gamma)$, implies $\gamma \alpha \leq \gamma \beta, \alpha \gamma \leq \beta \gamma$, for all $\gamma \in \Gamma$ and $\beta^{-1} \leq \alpha^{-1}$. Let $\Gamma$ be an ordered multiplicative group. A Krull valuation $\mid$ on ring $\mathrm{R}$ with values in $\Gamma$ is a mapping $\mid: R \rightarrow \Gamma \cup\{0\}$ satisfying the conditions.

(i) For $a \in R,|a|=0$ iff $a=0$;

(ii) For $a, b \in R,|a+b| \leq \operatorname{Max}\{|a|,|b|\}$;

(iii) For $a, b \in R,|a b|=|a||b|$.

with the properties $0.0=0,0 . \alpha=\alpha .0=0, \alpha \in \Gamma$ and $0<\alpha$ for all $\alpha \in \Gamma$.

Let $\Gamma$ be an ordered multiplicative group and $C_{1} \geq 1, C_{2} \geq 1$.

A $\left(C_{1}, C_{2}\right)$-Hölder Krull valuation on ring $\mathrm{R}$ with values in $\Gamma$ is a mapping $\|\|:. R \rightarrow \Gamma \cup\{0\}$ satisfying the conditions.

(i) For $a \in R,\|a\|=0$ iff $a=0$;

(ii) For $a, b \in R,\|a+b\| \leq C_{2} \operatorname{Max}\{\|a\|,\|b\|\}$;

(iii) For $a, b \in R, C_{1}^{-1}\|a\|\|b\| \leq\|a b\| \leq C_{1}\|a\|\|b\|$.

Remark. Note that $(1,1)$-Hölder Krull valuation on ring $\mathrm{R}$ is a classical Krull valuation on a ring $\mathrm{R}$.

In this paper $\mathrm{R}$ is a noncommutative ring with unit element.A ring $R^{\prime}$ is said to be a right ring of fraction if there is a given ring homomorphism $\varphi: R \rightarrow R^{\prime}$ such that. 
(a) $\varphi$ is S-inverting $(\varphi(S) \subset U(R)$, where $\mathrm{U}(\mathrm{R})$ is set of unit elements of $\mathrm{R})$.

(b) Every element of $R^{\prime}$ has the form $\varphi(a) \varphi(s)^{-1}$ for some $a \in R$ and $s \in S$.

(c) $\operatorname{Ker} \varphi=\{r \in R \mid r s=0$ for some $s \in S\}$.

The multiplicative set $S \subset R$ is right permutable if for any $a \in R$ and $s \in S, a S \cap s R \neq \emptyset$, also set $S \subset R$ is right reversible, for $a \in R$, if $s^{\prime} a=0$ for some $s^{\prime} \in S$, then $a s=0$ for some $s \in S$.

If the multiplicative set $S \subset R$ is both right permutable and right reversible, we shall say that $\mathrm{S}$ is a right denominator set. The ring $\mathrm{R}$ has a right ring of fractions with respect to multiplicative set $\mathrm{S}$ iff $\mathrm{S}$ is a right denominator set (see [5]).Let $\mathrm{S}$ be the multiplicative set of all regular elements. We say that $\mathrm{R}$ is a right ore ring iff $\mathrm{S}$ is right permutable, iff $R S^{-1}$ exists. In this case, we speak of $R S^{-1}$ as the classical right ring of quotients of $\mathrm{R}$, and denote it by $Q_{c l}^{r}(R)$. Let $\mathrm{R}$ be a domain and $S=R-\{0\}$. In this case, the right permutable condition on $\mathrm{S}$ may be re-expressed in the equivalent form: $a R \cap b R \neq 0$ for $a, b \in R-\{0\}$. This is called the (right) ore condition on $\mathrm{R}$. Thus, the domain $\mathrm{R}$ is right(resp. left) ore iff $\mathrm{R}$ satisfies the right (resp. left) ore condition.

\section{Krull valuation and $\left(C_{1}, C_{2}\right)$-Hölder Krull valuation for right ring of fractions}

Definition 1. Let $|\cdot|_{1}$ and $|\cdot|_{2}$ be two valuations on ring $R$. Then we say that $|\cdot|_{1}$ and $|\cdot|_{2}$ are $\left(C_{0}, \alpha\right)$-Hölder equivalent (where $C_{0} \geq 1, \alpha>0$ ) if for all $x \in R$,

$$
C_{0}^{-1}|x|_{1}^{\alpha^{\prime}} \leq|x|_{2} \leq C_{0}|x|_{1}^{\alpha^{\prime}}
$$

where $\alpha^{\prime}=\alpha$ or $\alpha^{\prime}=\alpha^{-1}$.

Lemma 1. Let $||:. R \rightarrow \Gamma \cup\{0\}$ be a Krull valuation on ring $R$ with right ring of fractions $R^{\prime}=Q_{\varphi}^{r}(R)$, where $\Gamma$ is an ordered multiplicative group. Then $|\cdot|_{\varphi}: R^{\prime} \rightarrow \Gamma \cup\{0\}$ by equation.

$$
|x|_{\varphi}=\left|\varphi(a) \varphi(b)^{-1}\right|_{\varphi}=|a||b|^{-1}, \text { for } a \in R, b \in S \text { is a Krull valuation on } \operatorname{ring} R^{\prime}=Q_{\varphi}^{r}(R)
$$

Proof. (i) Let $x \in R^{\prime}$ and $|x|_{\varphi}=\left|\varphi(a) \varphi(b)^{-1}\right|_{\varphi}=|a||b|^{-1}=0$ for some $a \in R, b \in S$. Then $|a|=0$ implies $a=0$. Hence $x=\varphi(0) / \varphi(b)=0$. Conversely, for $x \in R^{\prime}=Q_{\varphi}^{r}(R)$ if $x=0$, then $|x|_{\varphi}=|0|_{\varphi}=\left|\varphi(0) \varphi(1)^{-1}\right|=|0||1|^{-1}=0$.

(ii) For each $x, y \in R^{\prime}$, we have $|x+y|_{\varphi}=|\varphi(a) / \varphi(b)+\varphi(c) / \varphi(d)|_{\varphi}$ for some $a, c \in R$ and $b, d \in S$. From $b S \cap d R \neq \emptyset$, there exist $d_{1} \in S$ and $b_{1} \in R$ such that $b d_{1}=d b_{1}$. Thus,

$$
\begin{aligned}
|x+y|_{\varphi} & =\mid\left(\varphi(a) \varphi\left(d_{1}\right) /\left(\varphi(b) \varphi\left(d_{1}\right)\right)+\left(\varphi(c) \varphi\left(b_{1}\right) /\left.\left(\varphi(d) \varphi\left(b_{1}\right)\right)\right|_{\varphi}\right.\right. \\
& =\left|\varphi\left(a d_{1}+c b_{1}\right) / \varphi\left(b d_{1}\right)\right|_{\varphi} \\
& =\left|a d_{1}+c b_{1}\right|\left|b d_{1}\right|^{-1} \\
& =\left|a d_{1}+c b_{1}\right|\left|d b_{1}\right|^{-1} \\
& \leq \operatorname{Max}\left\{\left|a d_{1}\right|\left|b d_{1}\right|^{-1},\left|c b_{1}\right|\left|d b_{1}\right|^{-1}\right\} \\
& =\operatorname{Max}\left\{|a|\left|d_{1}\right|\left|d_{1}\right|^{-1}|b|^{-1},|c|\left|b_{1}\right|\left|b_{1}\right|^{-1}|d|^{-1}\right\} \\
& =\operatorname{Max}\left\{|a||b|^{-1},|c||d|^{-1}\right\} \\
& =\operatorname{Max}\left\{|x|_{\varphi},|y|_{\varphi}\right\}
\end{aligned}
$$

Hence $|x+y|_{\varphi} \leq \operatorname{Max}\left\{|x|_{\varphi},|y|_{\varphi}\right\}$. 
(iii) For each $x, y \in R^{\prime}$, we have $x y=(\varphi(a) / \varphi(b))(\varphi(c) / \varphi(d))$ for some $a, c \in R$ and $b, d \in S$. From $b R \cap c S \neq \emptyset$, we get elements $r \in R, s \in S$ such that $b r=c s \in S$, this implies that $c^{-1} b r=s$. Therefore, $d s=d c^{-1} b r, b^{-1} c=r s^{-1}$. Thus,

$$
x y=\varphi(a) \varphi\left(b^{-1}\right) \varphi(c) \varphi\left(d^{-1}\right)=\varphi(a) \varphi(r) \varphi\left(s^{-1}\right) \varphi\left(d^{-1}\right)=\varphi(a r)(\varphi(d s))^{-1} .
$$

Therefore,

$$
|x y|_{\varphi}=|\varphi(a r) / \varphi(d s)|_{\varphi}=|a r||d s|^{-1}=|a r|\left|d c^{-1} b r\right|^{-1}=|a||r||r|^{-1}|b|^{-1}|c||d|^{-1}=|a||b|^{-1}|c||d|^{-1}=|x|_{\varphi}|y|_{\varphi} .
$$

Consequently, $|\cdot|_{\varphi}$ is Krull valuation on $R^{\prime}=Q_{\varphi}^{r}(R)$.

Lemma 2. Let $||:. R \rightarrow \Gamma \cup\{0\}$ be a $\left(C_{1}, C_{2}\right)$-Hölder Krull valuation on ring $R$ with right ring of fractions $R^{\prime}=Q_{\varphi}^{r}(R)$, where $C_{1} \geq 1, C_{2} \geq 1$, and $\Gamma$ is an ordered multiplicative group. Then $|\cdot|_{\varphi}: R^{\prime}=Q_{\varphi}^{r}(R) \rightarrow \Gamma \cup\{0\}$ by equation: $|x|_{\varphi}=$ $\left|\varphi(a) \varphi(b)^{-1}\right|_{\varphi}=|a||b|^{-1}$ for $a \in R, b \in S$, is $\left(C_{1}^{4}, C_{1}^{2} C_{2}\right)$-Hölder Krull valuation on ring $R^{\prime}$.

Proof. (i) let $x \in R^{\prime}=Q_{\varphi}^{r}(R)$ and $|x|_{\varphi}=0$. Then $\left|\varphi(a) \varphi(b)^{-1}\right|_{\varphi}=|a||b|^{-1}=0$, for $a \in R, b \in S$. Therefore, $|a|=0$, it implies that $a=0$. Consequently, $x=\varphi(0) / \varphi(b)=0$. Conversely, let $x \in R^{\prime}=Q_{\varphi}^{r}(R)$ and $x=0$. Then

$$
|x|_{\varphi}=|0|_{\varphi}=\left|\varphi(0) \varphi(1)^{-1}\right|_{\varphi}=|0||1|^{-1}=0 .
$$

(ii) For each $x, y \in R^{\prime}=Q_{\varphi}^{r}(R)$, we have $|x+y|_{\varphi}=|\varphi(a) / \varphi(b)+\varphi(c) / \varphi(d)|$ for some $a, c \in R$ and $b, d \in S$. From $b S \cap d R \neq \emptyset$, there exist $d_{1} \in S$ and $b_{1} \in R$ such that $b d_{1}=d b_{1} \in S$. Thus, $\varphi(b) \varphi\left(d_{1}\right)=\varphi(d) \varphi\left(b_{1}\right)$. Therefore,

$$
\begin{aligned}
|x+y|_{\varphi} & =\left|\left(\varphi(a) \varphi\left(d_{1}\right)+\varphi(c) \varphi\left(b_{1}\right)\right) /\left(\varphi(b) \varphi\left(d_{1}\right)\right)\right|_{\varphi} \\
& =\mid \varphi\left(a d_{1}+c b_{1} /\left.\varphi\left(b d_{1}\right)\right|_{\varphi}=\left|a d_{1}+c b_{1}\right|\left|b d_{1}\right|^{-1}\right. \\
& \left.=\left|a d_{1}+c b_{1}\right|\left|d b_{1}\right|^{-1} \leq C_{2} \operatorname{Max}\left|a d_{1}\right|\left|b d_{1}\right|^{-1},\left|c b_{1}\right|\left|d b_{1}\right|^{-1}\right\} \\
& \leq C_{2} \operatorname{Max}\left\{C_{1}|a|\left|d_{1}\right| C_{1}\left|d_{1}\right|^{-1}|b|^{-1}, C_{1}|c|\left|b_{1}\right| C_{1}\left|b_{1}\right|^{-1}|d|^{-1}\right\} \\
& =C_{2} C_{1}^{2} \operatorname{Max}\left\{|a||b|^{-1},|c||d|^{-1}\right\}=C_{1}^{2} C_{2} \operatorname{Max}\left\{|x|_{\varphi},|y|_{\varphi}\right\} .
\end{aligned}
$$

(iii) For each $x, y \in R^{\prime}$, we have $x y=(\varphi(a) / \varphi(b))(\varphi(c) / \varphi(d))$, for some $a, c \in R$ and $b, d \in S$. From $b R \cap c S \neq \emptyset$, there exist $r \in R$ and $s \in S$ such that $b r=c s \in S$ implies $c^{-1} b r=s$. Therefore, $d s=d c^{-1} b r$ and $b^{-1} c=r s^{-1}$. Thus,

$$
x y=\varphi(a) \varphi\left(b^{-1}\right) \varphi(c) \varphi\left(d^{-1}\right)=\varphi(a) \varphi(r) \varphi\left(s^{-1}\right) \varphi\left(d^{-1}\right)=\varphi(a r)(\varphi(d s))^{-1} .
$$

Therefore,

$$
\begin{aligned}
|x y|_{\varphi} & =|\varphi(a r) / \varphi(d s)|_{\varphi}=|a r||d s|^{-1} \\
& =|a r|\left|d c^{-1} b r\right|^{-1} \geq C_{1}^{-1}|a||r|\left(C_{1}^{-1}|b r|^{-1}\left|d c^{-1}\right|^{-1}\right) \\
& \geq C_{1}^{-2}|a||r| C_{1}^{-1}|r|^{-1}|b|^{-1} C_{1}^{-1}|c||d|^{-1} \\
& \geq C_{1}^{-4}|a||b|^{-1}|c||d|^{-1}=C_{1}^{-4}|x|_{\varphi}|y|_{\varphi} .
\end{aligned}
$$

Thus,

$$
|x y|_{\varphi} \geq C_{1}^{-4}|x|_{\varphi}|y|_{\varphi} .
$$


Also, we have

$$
\begin{aligned}
|x y|_{\varphi} & =|a r|\left|d c^{-1} b r\right|^{-1} \leq C_{1}|a||r|\left(C_{1}|b r|^{-1}\left|d c^{-1}\right|^{-1}\right) \\
& \leq C_{1}^{2}|a||r| C_{1}|r|^{-1}|b|^{-1} C_{1}|c||d|^{-1} \\
& \leq C_{1}^{4}|a||b|^{-1}|c||d|^{-1}=C_{1}^{4}|x|_{\varphi}|y|_{\varphi} .
\end{aligned}
$$

Therefore,

$$
\left.\left.C_{1}^{-4}\right|_{x}\right|_{\varphi}|y|_{\varphi} \leq|x y|_{\varphi} \leq C_{1}^{4}|x|_{\varphi}|y|_{\varphi}
$$

Consequently, $|\cdot|_{\varphi}$ is $\left(C_{1}^{4}, C_{1}^{2} C_{2}\right)$-Hölder Krull valuation on $\operatorname{ring} R^{\prime}=Q_{\varphi}^{r}(R)$.

Definition 2. Let $R$ be a ring. We say that $R$ satisfies in Hölder rigidity condition if for every $\left(C_{1}, C_{2}\right)$-Hölder Krull valuation $|$.$| on R$, there exists a classical Krull valuation $\|$.$\| on R$ such that $\|\cdot\|$ is $\left(C_{0}, \alpha\right)$-Hölder equivalent (where $C_{0} \geq 1, \alpha>0$ ) to $|$.$| .$

Theorem 1. Let $R$ be a ring satisfying in Hölder rigidity condition, and $S$ a right permutable set of regular elements in $R$. Then the right ring of fractions $R^{\prime}=Q_{\varphi}^{r}(R)$ with respect to $S$ satisfies in Hölder rigidity condition.

Proof. Let $\|\cdot\|_{\varphi}: R^{\prime} \rightarrow \Gamma \cup\{0\}$ be $\left(C_{1}, C_{2}\right)$-Hölder Krull valuation on right ring of fractions $R^{\prime}=Q_{\varphi}^{r}(R)$, where $\Gamma$ is an ordered abelian multiplicative group, $C_{1} \geq 1$ and $C_{2} \geq 1$. We define $\|\cdot\|: R \rightarrow \Gamma \cup\{0\}$ by equation: $\|a\|=\|\varphi(a)\| \varphi$, for all $a \in R$. Thus, we have.

(i) let $a \in R$ and $a=0$. Then $\|0\|=\|\varphi(0)\|_{\varphi}=\|0\|_{\varphi}=0$. Conversely, let $a \in R$ and $\|a\|=0$. Then $\|\varphi(a)\|_{\varphi}=0$ implies $\varphi(a)=0$. Hence there exists $s \in S$ such that $a s=0$. Therefore, $a=0$ (s is regular element).

(ii) For each $a, b \in R$, we have

$$
\|a+b\|=\|\varphi(a+b)\|_{\varphi}=\|\varphi(a)+\varphi(b)\|_{\varphi} \leq C_{2} \operatorname{Max}\left\{\|\varphi(a)\|_{\varphi},\|\varphi(b)\|_{\varphi}\right\}=C_{2} \operatorname{Max}\{\|a\|,\|b\|\} .
$$

(iii) For each $a, b \in R$, we have

$$
C_{1}^{-1}\|a\|\|b\|=C_{1}^{-1}\|\varphi(a)\|_{\varphi}\|\varphi(b)\|_{\varphi} \leq\|\varphi(a) \varphi(b)\|_{\varphi}\left(=\|\varphi(a b)\|_{\varphi}=\|a b\|\right) \leq C_{1}\|\varphi(a)\|_{\varphi}\|\varphi(b)\|_{\varphi}=C_{1}\|a\|\|b\| .
$$

Therefore, $\|\cdot\|$ is $\left(C_{1}, C_{2}\right)$-Hölder Krull valuation on R. Since R satisfies in Hölder rigidity condition, hence there exists a classical Krull valuation |.| on $\mathrm{R}$ such that $\left(C_{0}, \alpha\right)$-Hölder equivalent (where $\left.C_{0} \geq 1, \alpha>0\right)$ to $\left(C_{1}, C_{2}\right)$-Hölder Krull valuation $\|$.$\| on ring \mathrm{R}$. Now by Lemma 2 , the mapping

$$
|\cdot|_{\varphi}: R^{\prime}=Q_{\varphi}^{r}(R) \rightarrow \Gamma \cup\{0\} \quad \text { by } \quad|x|_{\varphi}=\left|\varphi(a) \varphi(b)^{-1}\right|_{\varphi}=|a||b|^{-1}
$$

( for $a \in R, b \in S$ ) is a Krull valuation on $\operatorname{ring} R^{\prime}=Q_{\varphi}^{r}(R)$. On the other hand, for each $a \in R$, we have

$$
C_{0}^{-1}|a|^{\alpha^{\prime}} \leq\|a\| \leq C_{0}|a|^{\alpha^{\prime}}
$$


where $\alpha^{\prime}=\alpha$ or $\alpha^{\prime}=\alpha^{-1}$. Therefore, for each $x=\varphi(a) / \varphi(b)$, for some $a \in R, b \in S$, we have

$$
\begin{aligned}
\|x\|_{\varphi} & =\left\|\varphi(a) \varphi(b)^{-1}\right\|_{\varphi} \leq C_{1}\|\varphi(a)\|_{\varphi}\|\varphi(b)\|_{\varphi}^{-1}\left(=C_{1}\|a\|\|b\|^{-1}\right) \\
& \leq C_{1} C_{0}|a|^{\alpha^{\prime}} C_{0}|b|^{-\alpha^{\prime}}\left(=C_{1} C_{0}^{2}\left(|a||b|^{-1}\right)^{\alpha^{\prime}}=C_{1} C_{0}^{2}|x|_{\varphi}^{\alpha^{\prime}}\right) .
\end{aligned}
$$

on the other hand,

$$
\begin{aligned}
\|x\|_{\varphi} & =\left\|\varphi(a) \varphi(b)^{-1}\right\|_{\varphi} \geq C_{1}^{-1}\|\varphi(a)\|_{\varphi}\|\varphi(b)\|_{\varphi}^{-1}\left(=C_{1}^{-1}\|a\|\|b\|^{-1}\right) \\
& \geq C_{1}^{-1} C_{0}^{-1}|a|^{\alpha^{\prime}} C_{0}^{-1}\left(|b|^{-1}\right)^{\alpha^{\prime}}=C_{1}^{-1} C_{0}^{-2}\left(|a \| b|^{-1}\right)^{\alpha^{\prime}}=C_{1}^{-1} C_{0}^{-2}|x|_{\varphi}^{\alpha^{\prime}} .
\end{aligned}
$$

Therefore,

$$
\left(C_{1} C_{0}^{2}\right)^{-1}|x|_{\varphi}^{\alpha^{\prime}} \leq\|x\|_{\varphi} \leq C_{1} C_{0}^{2}|x|_{\varphi}^{\alpha^{\prime}}
$$

Hence $|.|_{\varphi}: R^{\prime} \rightarrow \Gamma \cup\{0\}$ is $\left(C_{1} C_{0}^{2}, \alpha\right)$-Hölder equivalent to $\left(C_{1}, C_{2}\right)$-Hölder Krull valuation $\|\cdot\|_{\varphi}$ on ring $R^{\prime}$. Therefore, $R^{\prime}$ satisfies in Hölder rigidity condition.

\section{Competing interests}

The authors declare that they have no competing interests.

\section{Authors' contributions}

All authors have contributed to all parts of the article. All authors read and approved the final manuscript.

\section{References}

[1] O. Endler, Valuation Theory, New York, Springer-Verlag, (1972).

[2] E. Munoz Garcia, Hölder absolute values are equivalent to classicalones, Proc. Amer. Math. Soc, 127(7), 1967-1971, (1999).

[3] J. A. Huckaba, Commutative Rings with Zero Divisors, New York, Marcel Dekker, Inc (1988).

[4] M. Knebusch, D. Zhang, Manis Valuations and Prufer Extensions, I. A new chapter in commutative algebra. Lecture Notes in Math. 1791. Berlin, Springer-Verlage, (2002).

[5] T. Y. Lam, A First Course in Noncommutative Rings, Springer-Verlag, (1991).

[6] M. Manis, Valuations on a commutative ring, Proc. Amer. Math. Soc, 20, 193-198, (1969).

[7] P. Ribenboim, The theorie des Valuations, Montreal, Less presses de I Universite de Montreal (1964).

[8] O. F. G. Schilling, The theory of Valuations, New York, Amer. Math. Soc, Thomas, J. J, (1978). Set theory, New York, Academic press, Inc (1952). 